\title{
Tratamiento quirúrgico de fístula arteriovenosa pulmonar en telangectasia hemorrágica hereditaria (Enfermedad de Rendu Osler Weber)*
}

\author{
Drs. ROBERTO GONZÁLEZ L. ${ }^{1,2}$, DAVID LAZO P. ${ }^{1,2}$, RAFAEL PRATS M. ${ }^{1,2}$, \\ RAIMUNDO SANTOLAYA C. ${ }^{1,2}$, VIRGINIA LINACRE S. ${ }^{1}$, PATRICIO RODRÍGUEZ D. ${ }^{1,2}$ \\ 1 Sección de Cirugía de Tórax, Servicio Médico Quirúrgico Respiratorio, Instituto Nacional del Tórax. \\ 2 Departamento de Cirugía, Campus Oriente, Universidad de Chile. \\ Santiago, Chile.
}

\begin{abstract}
Pulmonary arteriovenous fistula in hereditary hemorrhagic telangiectasia. Report of one case
\end{abstract}

Hereditary hemorrhagic telangiectasia (Osler-Weber-Rendu syndrome) is an autosomal dominant vascular disease that can be associated with pulmonary arteriovenous fistulas. We report a 16 years old female with an Osler-Weber-Rendu syndrome and a history of frequent episodes of epistaxis since the age of 10 years. A Chest CAT scan performed on that occasion showed an arteriovenous fistula. The patient presented with progressive dyspnea, low arterial oxygen saturation and clubbing of fingers. An echocardiogram and lung perfusion scintigram confirmed the presence a right to left shunt. The patient was subjected to a lobectomy with a good postoperative evolution.

Key words: Arteriovenous fistula, Osler-Weber-Rendu syndrome, dyspnea.

\section{Resumen}

Las Fístulas Arteriovenosas Pulmonares (FAVP son malformaciones vasculares infrecuentes. La Telangectasia Hemorrágica Hereditaria o Enfermedad de Rendu-Osler-Weber (EROW) es una enfermedad hereditaria autosómica dominante que se asocia con frecuencia a dichas malformaciones vasculares pulmonares. Presentamos el caso de una mujer de 16 años con EROW y una FAVP única, sintomática y que comprometía extensamente el lóbulo medio, en quien realizamos tratamiento con cirugía resectiva pulmonar (lobectomía media) y que evolucionó satisfactoriamente con desaparición de la sintomatología.

Palabras clave: Telangiectasia hemorrágica hereditaria, malformaciones arteriovenosas, pulmón, cirugía torácica, Rendu Osler Weber.

*Recibido el 28 de Julio de 2009 y aceptado para publicación el 19 de Noviembre de 2009.

No existen conflictos de interés ni apoyo financiero.

Correspondencia: Dr. Roberto González L.

José Manuel Infante 717, Santiago, Chile.

Fax: 056-02-5754997

E-mail: rgonzalezlagos@udec.cl 


\section{Introducción}

Las Fístulas Arteriovenosas Pulmonares (FAVP) son malformaciones vasculares infrecuentes y corresponden a una comunicación anómala entre una arteria y una vena pulmonar. La mayoría de las FAVP son de origen congénito y su incidencia es de 2 a 3 casos por cada 100.000 habitantes ${ }^{1-3}$. Estas fístulas producen un "shunt" de derecha a izquierda cuya magnitud es variable y está determinado principalmente por el tamaño de la fístula ${ }^{1-3}$.

La Telangectasia Hemorrágica Hereditaria o Enfermedad de Rendu-Osler-Weber (EROW) es una enfermedad hereditaria autosómica dominante que se asocia con frecuencia a dichas malformaciones vasculares pulmonares ${ }^{4,5}$.

Presentamos el caso de una mujer de 16 años con EROW y una FAVP única, sintomática y que comprometía extensamente el lóbulo medio, en quien realizamos tratamiento con cirugía resectiva pulmonar.

\section{Caso Clínico}

Mujer de 16 años quien a $\operatorname{los} 10$ años presentó un accidente neurológico secundario a una Malformación Arteriovenosa (MAV) cerebral parietal derecha. Fue tratada con embolización y evolucionó sin secuelas neurológicas. En la anamnesis relataba episodios frecuentes de epistaxis y al examen físico presentaba telangectasias faciales. Como antecedentes familiares destacaba que la paciente tenía un hermano mayor sin antecedentes de importancia, su abuelo paterno falleció de una epistaxis exanguinante, su padre y el hermano de éste presentaban telangectasias mucocutáneas y epistaxis a repetición. En este contexto clínico y familiar se diagnosticó una EROW.

En el estudio de búsqueda de otras MAV la radiografía de tórax mostró una lesión en el lóbulo medio que en la tomografía computada de tórax era compatible con una fístula arteriovenosa. Se mantuvo en controles periódicos con su pediatra tratante. Luego del desarrollo puberal se hizo sintomática con cianosis, disnea progresiva a los esfuerzos (capacidad funcional III) e intolerancia al frío; al examen físico destacaban telangectasias faciales y marcada acropaquia. La saturación de $\mathrm{O}_{2}$ en reposo era de $76 \%$.

Entre los exámenes de laboratorio destacó un hematocrito de $50 \%$ y una hemoglobina de $18 \mathrm{~g} / \mathrm{dl}$. La radiografía de tórax (Figura 1) y la tomografía computada de tórax (Figura 2A, 2B) mostraron la lesión en el lóbulo medio compatible con una fístula arteriovenosa de gran tamaño. El ecocardiograma confirmó el "shunt" y no evidenciaba hipertensión pulmonar ni dilatación de cavidades cardíacas. El cintigrama de perfusión pulmonar con Tc. 99-MAA era compatible con un shunt de derecha a izquierda con índice de actividad del 53\%.

En este contexto fue derivada a nuestro equipo de cirugía. Debido a que la fístula arteriovenosa era única y comprometía extensamente el lóbulo medio se planteó cirugía resectiva pulmonar. En el intra operatorio se encontró una MAV que comprometía gran parte del lóbulo medio, destacaba una importante dilatación de las venas y arterias lobares; no se encontraron otros hallazgos significativos. Se realizó la lobectomía media sin incidentes. En el post operatorio inmediato se observó una mejoría significativa en la oximetría de pulso que alcanzó al 99\%. La paciente evolucionó satisfactoriamente y fue dada de alta hospitalaria al cuarto día post operatorio. El estudio de anatomía patológica confirmó la presencia de una fístula arteriovenosa pulmonar que comprometía gran parte del lóbulo medio. A 9 meses de seguimiento se encuentra en controles periódicos con médicos tratantes, sin cianosis, sin disnea (capacidad funcional I), sin intolerancia al frío, sin acropaquia y con saturación de $\mathrm{O}_{2}$ de $99 \%$.

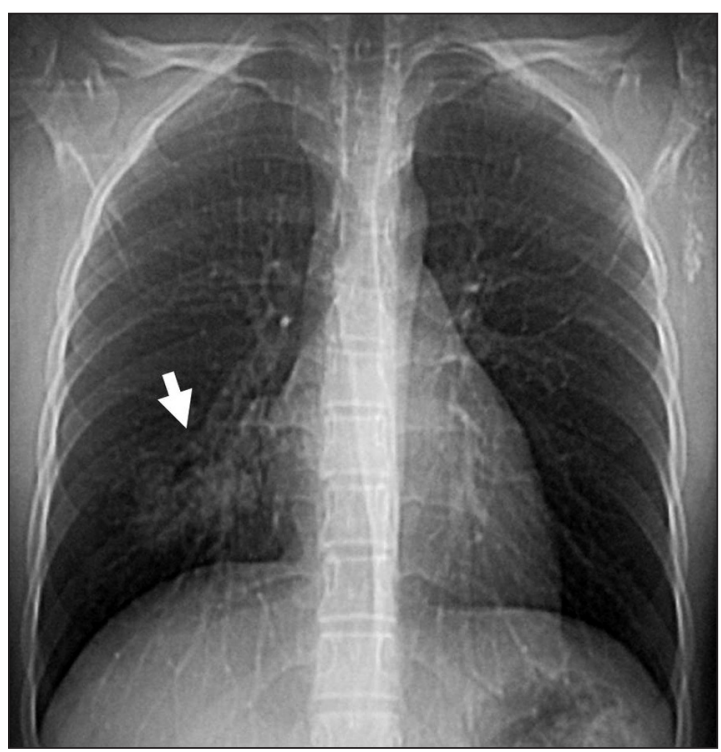

Figura 1. Radiografía de tórax: se observa la lesión en lóbulo medio (flecha). 


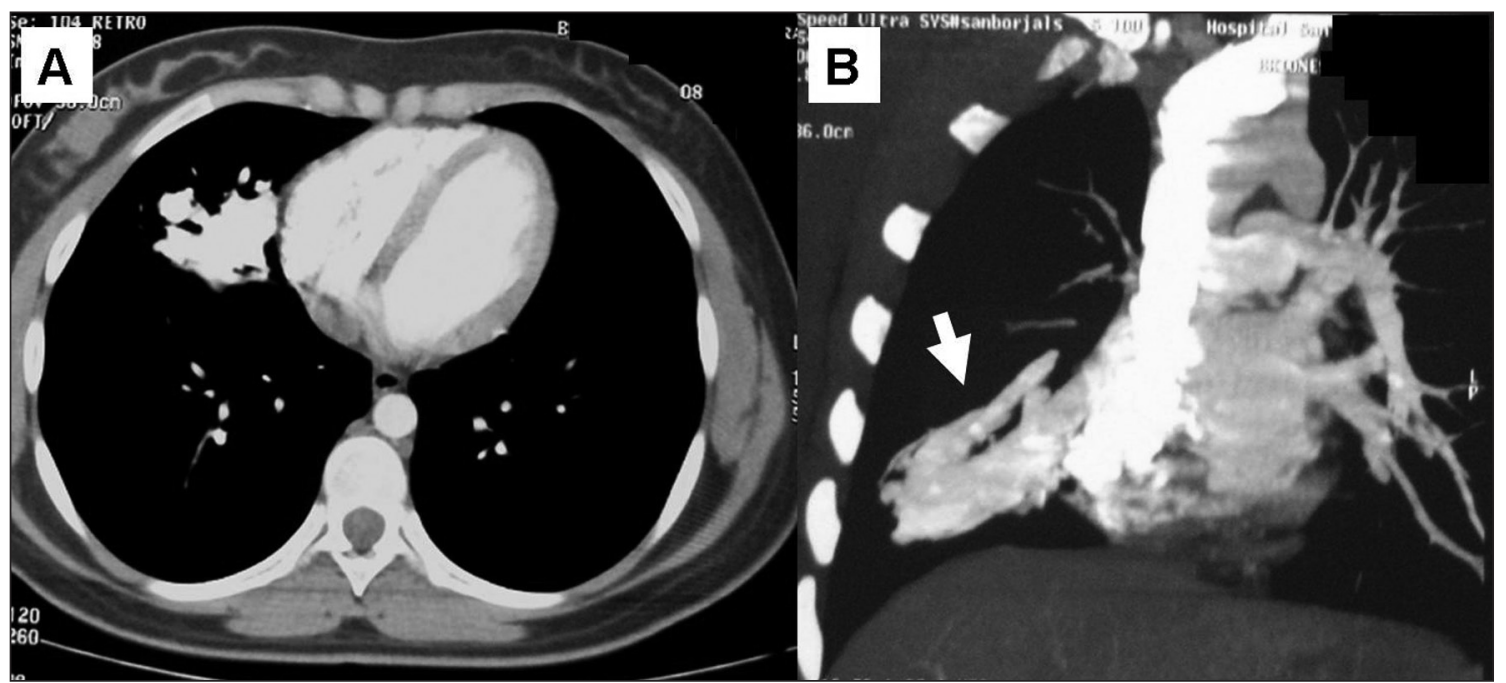

Figura 2. Tomografía computada de tórax en que se observa malformación arteriovenosa. A. Corte axial: se observa compromiso de lóbulo medio. B. Reconstrucción: se observa extenso lecho vascular de fístula arteriovenosa en lóbulo medio (flecha).

\section{Discusión}

Las Fístulas Arteriovenosas Pulmonares (FAVP) son más frecuentes en el género femenino (relación 2:1), entre el 65 a $70 \%$ de los casos se presentan en pacientes con Enfermedad de Rendu-Osler-Weber (EROW), por otro lado entre el 15 al $35 \%$ de los pacientes con EROW presentan FAVP ${ }^{1-6}$.

La EROW es una enfermedad de origen genético que se caracteriza por epistaxis recurrente, telangectasias mucocutáneas y malformaciones vasculares viscerales (pulmón, cerebro, hígado, páncreas, etc. $)^{4,5}$. Las FAVP en EROW frecuentemente se encuentran en los lóbulos inferiores (60-95\%), pueden ser múltiples (35-65\%), uni o bilaterales y no existe predominio de lado ${ }^{1-6}$.

En estos pacientes las fístulas se manifiestan clínicamente a nivel pulmonar generalmente después de la tercera década de la vida y principalmente entre la quinta y sexta, ya que las manifestaciones clínicas en las primeras décadas son frecuentemente la epistaxis y las telangectasias mucocutáneas ${ }^{4,5}$.

A nivel pulmonar las manifestaciones clínicas de las FAVP en EROW son: hipoxemia como consecuencia del "shunt" de derecha a izquierda, lo que produce disnea, cianosis y policitemia; embolias paradójicas sistémicas especialmente a nivel cerebral (abscesos y accidentes isquémicos cerebrales) y sangrado (hemoptisis y hemotórax) por rotura ${ }^{6}$. La hemoptisis y el hemotórax espontáneo masivo pueden ser una forma infrecuente de presentación, lo que ha sido principalmente descrito en mujeres embarazadas en quienes el tamaño de la fístula experimenta un importante aumento ${ }^{4-10}$.

La radiografía de tórax es anormal hasta en el 98\% de los casos, se observan masas ovaladas especialmente en lóbulos inferiores únicas o múltiples, uni o bilaterales ${ }^{3,6}$. La tomografía computada helicoidal ha desplazado a la angiografía tradicional en el diagnóstico de las FAVP en EROW, permite evaluar ubicación, tamaño, vasos aferentes y eferentes. El uso de medio de contraste y las reconstrucciones tridimensionales permiten una excelente evaluación anatómica de las lesiones, así como evaluar otras lesiones intratorácicas ${ }^{1,6,11}$. El diagnóstico ecocardiográfico con contraste (burbujas) es muy útil especialmente en la pesquisa de las FAVP, permite objetivar el "shunt" y evaluar la presencia y cuantía de hipertensión pulmonar. El cintigrama de perfusión pulmonar es excelente para cuantificar adecuadamente el "shunt"

El tratamiento se recomienda en las FAVP sintomáticas o mayores de 3 milímetros ${ }^{1-6}$. La embolización ha demostrado ser segura y obtener buenos resultados con las ventajas propias de los tratamientos percutáneos ${ }^{4-6}$. La cirugía resectiva continúa teniendo un rol en los casos en que la embolización falla o no es posible realizar, en cirugías de urgencia por rotura y sangrado, o en las FAVP de gran tamaño como en nuestra paciente, en que la lesión era única y comprometía extensamente el lóbulo medio ${ }^{1,12-17}$.

La EROW se asocia a FAVP que pueden presentarse en un amplio espectro clínico y anatómico, pueden generar importantes síntomas y graves 
complicaciones por lo que en general se recomienda su tratamiento. La cirugía resectiva pulmonar está indicada en algunos casos seleccionados.

\section{Referencias}

1. Pick A, Deschamps C, Stanson AW. Pulmonary arteriovenous fistula: presentation, diagnosis, and treatment. World J Surg 1999; 23: 1118-1122.

2. Khurshid I, Downie GH. Pulmonary arteriovenous malformation. Postgrad Med J 2002; 78: 191-197.

3. López Vime R, de Miguel Díez J, Jara Chinarro B, Salgado Salinas R, Gómez Santos D, Serrano Iglesias JA. Diagnosis and treatment of pulmonary arteriovenous fistulas. Arch Bronconeumol 2002; 38: 288-290.

4. Sharathkumar AA, Shapiro A. Hereditary haemorrhagic telangiectasia. Haemophilia 2008; 14: 1269-1280.

5. Begbie ME, Wallace GM, Shovlin CL. Hereditary haemorrhagic telangiectasia (Osler-Weber-Rendu syndrome): a view from the 21st century. Postgrad Med J 2003; 79: 18-24.

6. Cottin V, Dupuis-Girod S, Lesca G, Cordier JF. Pulmonary vascular manifestations of hereditary hemorrhagic telangiectasia (Rendu-Osler Disease). Respiration 2007; 74: 361-378.

7. Litzler PY, Douvrin F, Bouchart F, Tabley A, Lemercier $\mathrm{E}$, Baste JM et al. Combined endovascular and videoassisted thoracoscopic procedure for treatment of a ruptured pulmonary arteriovenous fistula: Case report and review of the literature. J Thorac Cardiovasc Surg 2003; 126: 1204-1207.

8. Ausín Herrero P, Gómez-Caro Andrés A, Moradiellos Díez FJ. Spontaneous hemothorax due to rupture of a pulmonary artery aneurysm in Rendu-Osler-Weber síndrome. Arch Bronconeumol 2004; 40: 603.

9. Khan AA, Hunt I, Hamdane K, Tambiah J, Deshpande RP, Reidy JF. Massive pulmonary arteriovenous malformation presenting with tamponading haemothorax. Thorax 2007; 62: 836 .

10. Swietlik E, Doboszynska A. Recurrence of arterio-venous malformations with life-threatening complications in a pregnant woman with hereditary teleangiectasia. J Physiol Pharmacol 2008; 59 Suppl 6: 683-688.

11. $\mathrm{Hu} \mathrm{SY}$, Tsai CH, Tsan YT. Pulmonary arteriovenous malformation in Osler-Weber-Rendu syndrome. Eur J Cardiothorac Surg 2009; 36: 395.

12. Temes RT, Paramsothy P, Endara SA, Wernly JA. Resection of a solitary pulmonary arteriovenous malformation by video- assisted thoracic surgery. J Thorac Cardiovasc Surg 1998; 116: 878-879.

13. Schröder C, Fröhlich G, Harms CP, Kleckow M, Macchiarini P. Fistulectomy as an alternative to segmentectomy for pulmonary arteriovenous fistula. J Thorac Cardiovasc Surg 2001; 122: 386-388.

14. Georghiou GP, Berman M, Vidne BA, Saute M. Pulmonary arteriovenous malformation treated by lobectomy. Eur J Cardiothorac Surg 2003; 24: 328-330.

15. Lischke R, Simonek J, Stolz A, Pafko P. Bilateral pulmonary arteriovenous malformations in patient with Rendu-Osler-Weber disease. Eur J Cardiothorac Surg 2004; 25: 461.

16. Fraga JC, Favero E, Contelli F, Canani F. Surgical treatment of congenital pulmonary arteriovenous fistula in children. J Pediatr Surg 2008; 43: 1365-1367.

17. Takahama M, Yamamoto R, Nakajima R, Izumi N, Tada H. Surgery for multiple and diffuse pulmonary arteriovenous fistulas during childhood. Gen Thorac Cardiovasc Surg 2009; 57: 385-388. 